\title{
ISW for the Treatment of Adult Facial Asymmetry and Tooth Crowding
}

Chang Yuan-Chieh, YU Jian-Hong, Tsai Ya-Yu, YU Chien-Chih

\begin{abstract}
Improved superelastic Ti-Ni alloy wire (ISW) was used for the management of a 24-year-old patient presenting with facial asymmetry and maxillary and mandibular anterior dental crowding. After the extraction of teeth \#14, \#24, \#34, \#45, ISW leveling was performed to relieve upper and lower anterior crowding. Elastic chain and intermaxillary elastics (IME) were then used to correct the mandibular shift. The correction of facial asymmetry was successfully achieved within 31 months. The outcome was the accomplishment of an esthetic smiling arc that achieved patient satisfaction after the completion of orthodontic treatment.
\end{abstract}

Keywords: Improved superelastic $\mathrm{Ti}-\mathrm{Ni}$ alloy wire, Intermaxillary elastics, Facial asymmetry, Dental crowding.

How to cite this article: Yuan-Chieh C, Jian-Hong YU, Ya-Yu T, Chien-Chih YU. ISW for the Treatment of Adult Facial Asymmetry and Tooth Crowding. Int J Experiment Dent Sci 2013;2(2):134-138.

Source of support: This work was supported by China Medical University and Hospital, Taichung City, Taiwan (Grant number: CMU95-120).

\section{Conflict of interest: None}

\section{INTRODUCTION}

Improved superelastic Ti-Ni alloy wire (ISW) was developed by Tokyo Medical and Dental University and marketed under the brand name of L and $\mathrm{H}$ Titan wire. ISW offers three main significant properties characterized by superelasticity, shape memory, and shock and vibration absorbing capacity. ${ }^{1}$ The wire can be easily applied in the slots of the brackets that are away from the dental arch, and can be restored to its original form after being taken from the brackets. The vibration absorbing property enables it to buffer the force transmission to the periodontal ligament, which reduces the discomfort and potential risk of root resorption for the patients receiving orthodontic treatment. ${ }^{2,3}$

The facial midline is taken as the dividing line from glabella, passing from subnasale to menton. Facial asymmetry refers to a lack of symmetry comparing two parts of the face in shape, size and relative position. The etiology of facial asymmetry includes genetic imperfections, pathological problems or functional displacement. ${ }^{4}$ Genetics have been implicated in some diseases like multiple neurofibromatosis that is associated with a autosomal dominant genetic disorder. ${ }^{5}$ Pathological problems may be related to tumors, infection, condylar resorption and condylar fractures. ${ }^{6}$ Functional shift can result from the presence of occlusal interferences such as malposed teeth, missing teeth or improper size teeth. Thus, before correcting the asymmetrical facial problem, the other interferences should be treated in the first place and the goal of facial asymmetry correction should consist of both occlusal correction and alignment of dental midline.

This article describes the orthodontic management of a woman with facial asymmetry and dental crowding, in which the ISW, elastic chains, intermaxillary elastics (IME), and some smart strategies were used for treatment.

\section{DIAGNOSIS AND TREATMENT PLAN}

A 24-year-old female with a chief complaint of poor dental occlusion sought treatment at the Department of Orthodontics. The patient had no history of disease, drug allergy, bad habits or temporomandibular joint (TMJ) problem. Pretreatment cephalometric and posteroanterior $\mathrm{X}$-ray films revealed that the patient presented with an Angle class I malocclusion and low angle of the mandibular arch shifted to the right side (Figs 1A to C). In addition, crowding in the maxillary and mandibular anterior teeth was observed. The treatment plan involved the extraction of 7 teeth, including $\# 14, \# 24, \# 34, \# 45, \# 18, \# 28, \# 38$, followed by the combined use of metal bracket*, clear bracket ${ }^{*}$ for direct bonding to teeth. All spaces of the arch were successfully closed and aligned by using archwire* (TOMY L\& $\mathrm{H}^{\circledR}$ Titan Super Elastic Nickel Titanium Alloy wire, $0.016^{\prime \prime} \times 0.022^{\prime \prime}$ Accu Form $\left.{ }^{\circledR}\right)$, closed coil spring* (50 gf, 100 gf), elastic chain* and IME** (medium, 3/16 inch size).

\section{Treatment Progress}

At the beginning of treatment, the archwire was fully engaged in the brackets for leveling and decrowding the teeth. The orthodontic spaces of \#14, \#24, \#34, \#45, and extraction position were closed by using closed coil springs (100 gf) that generated a canine distal driving force to retract

\footnotetext{
* Super chain (Elastic chain), Tomy incorporated, 3-16-7 Midoricho, Fuchucity, Tokyo, 183-0006 Japan; www.tomyinc.co.jp **3M Unitek, 6F., No. 95, Sec. 2, Dunhua S. Rd., Da'an Dist., Taipei City 106, Taiwan (R.O.C.); solutions.3m.com.tw
} 

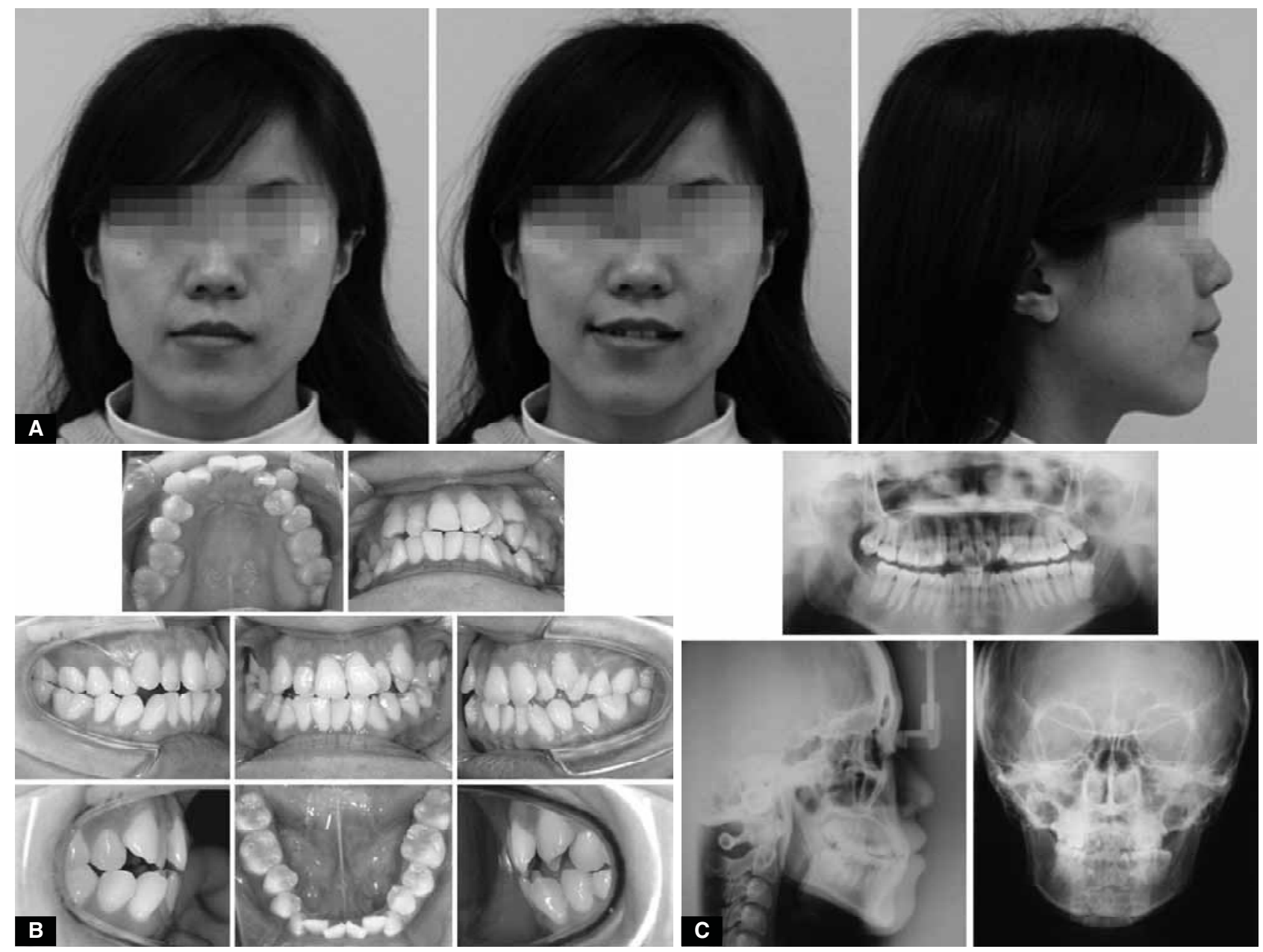

Figs 1 A to $\mathbf{C}$ : The images of the patient showing that the upper and lower anterior teeth are crowding, and the mandible is shifted to the right side

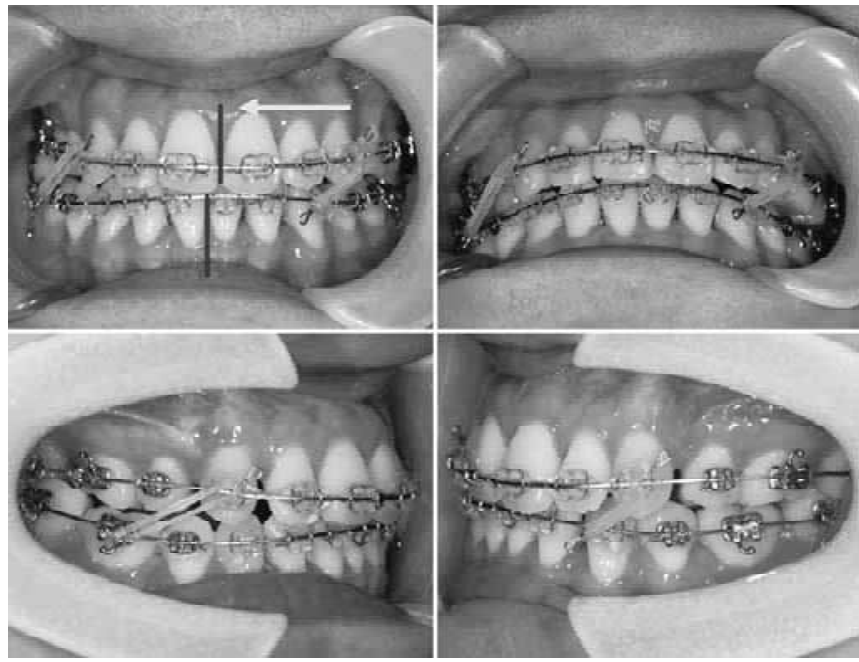

Fig. 2: Elastic chain was used to move the teeth \#21, \#22, and \#23 to the left side, and IME was used between the teeth \#13, \#46 and \#23, \#33 for upper teeth dental midline correction

the anterior teeth. To solve the problem of facial asymmetry, elastic chain and IME were used to correct the dental midline. In the upper arch, we intentionally shifted the left teeth to the right side (Fig. 2). In contrast, the shift was made to the opposite side in the lower arch (Fig. 3). At the

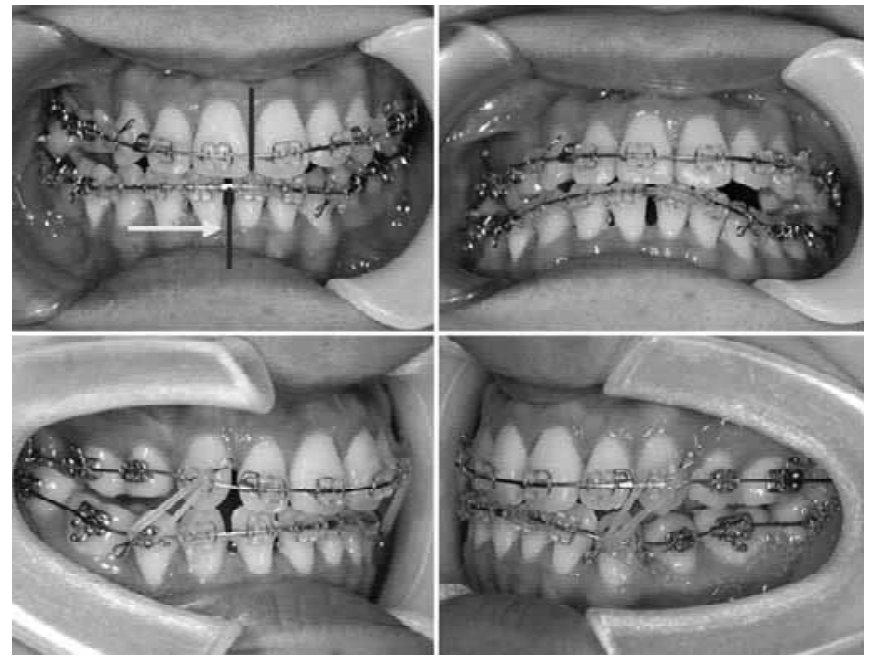

Fig. 3: Elastic chain was used to move the teeth \#41, \#42, and \#43 to the left side, and IME was used between the teeth \#13, \#44 and \#23, \#33 for lower teeth dental midline correction

end of treatment, traditional retainers and dynamic positioner were used as retentive devices for more detailed alignment of the teeth. The active orthodontic treatment required a total of 31 months to complete, and correction was successfully achieved (Figs 4 and 5). 

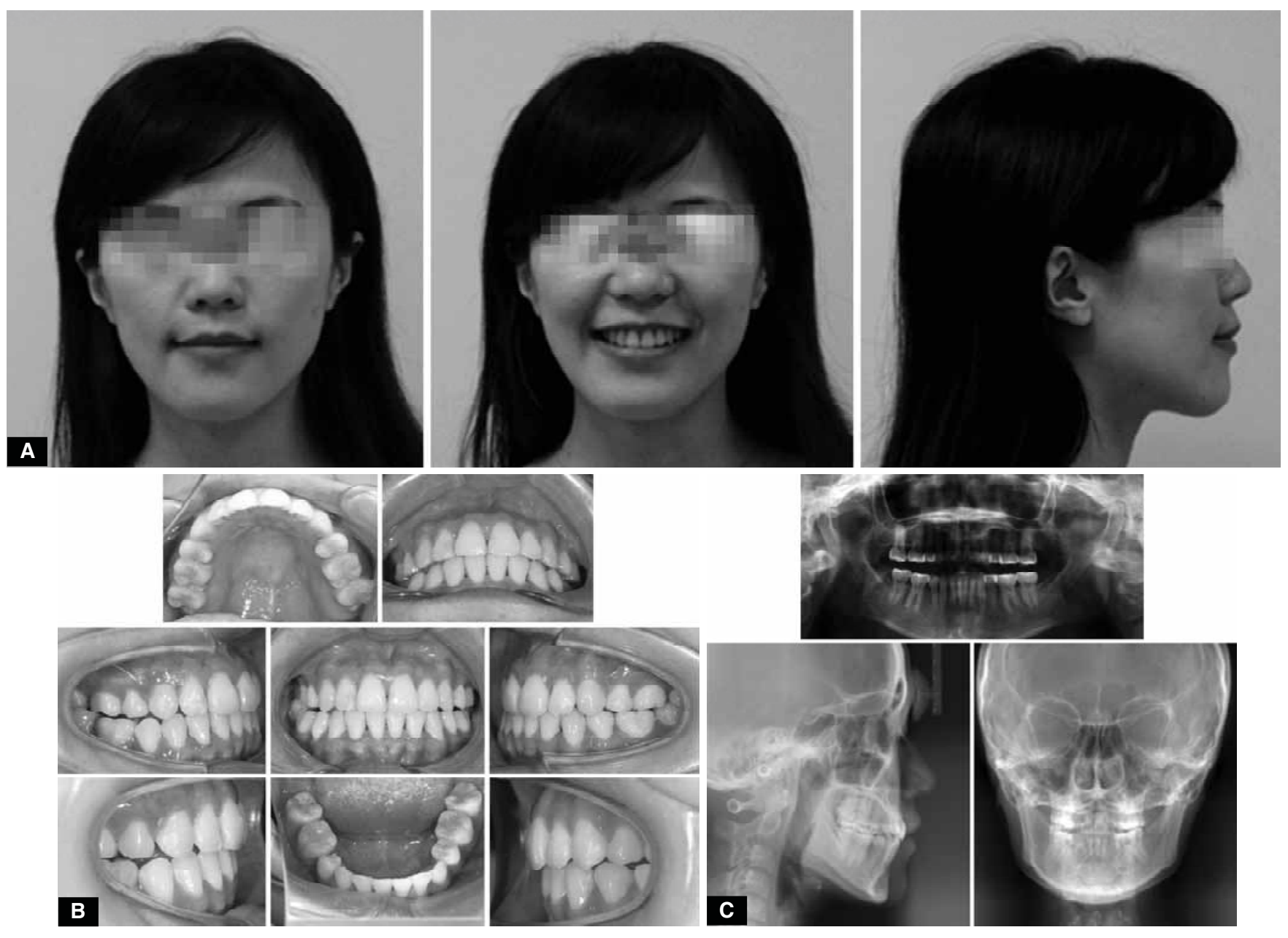

Figs 4A to C: The facial asymmetry problem was successfully improved after the active orthodontic treatment
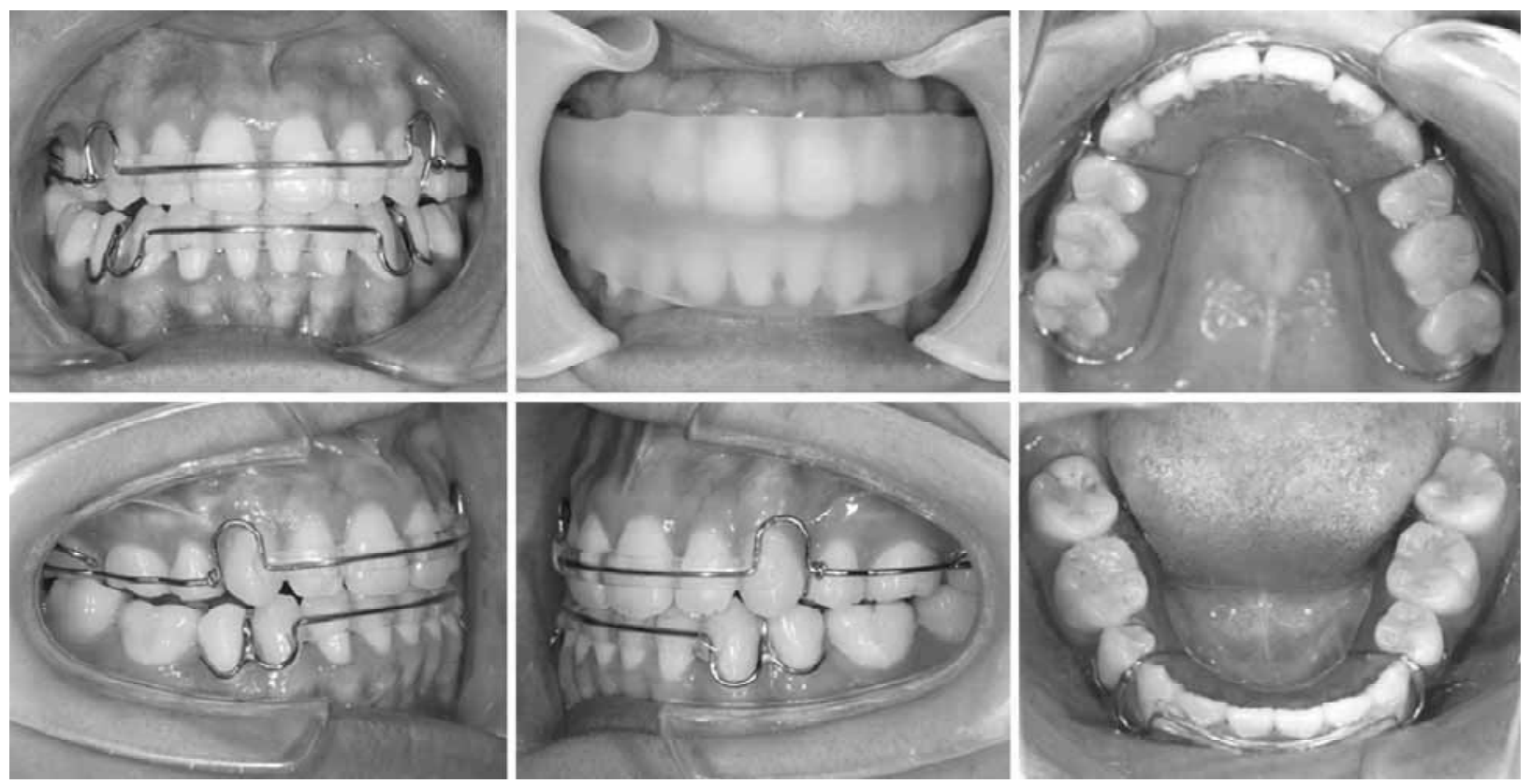

Fig. 5: Traditional retainers and dynamic positioner were delivered to maintain the jaw relationship after active orthodontic treatment

\section{DISCUSSION}

The methods to resolve dental crowding include flaring of incisors, expansion of the arches, distalization of molars, extraction of teeth and stripping. Extraction of teeth is not a necessary approach for all cases with crowded teeth; some patients only need the teeth to be leveled. The considerations 

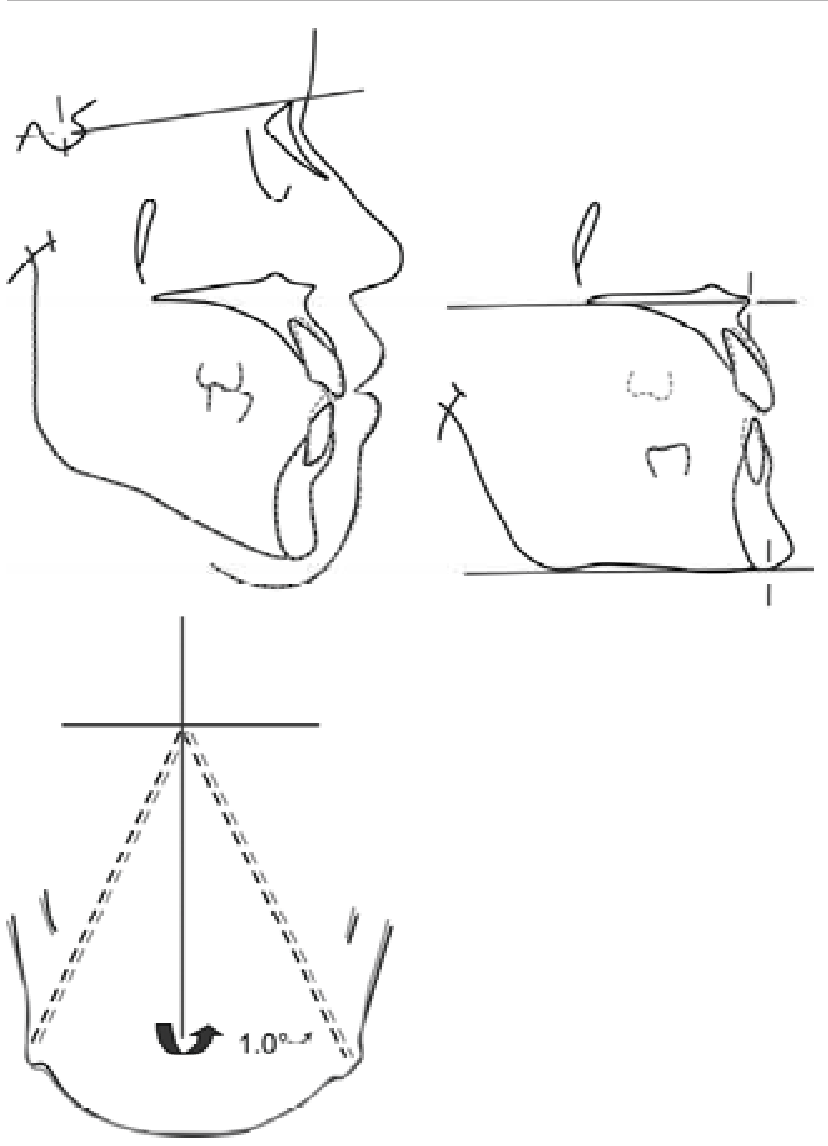

Fig. 6: The superimposition drawings represent the pretreatment profile in dark line, and the post-treatment profile in dotted line. Note that mandibular plane angle is mildly increased and the mandible is shifted to the left side and decision can be focused on the facial profile, the condition of the teeth, and the mandibular plane angle of patient, as well as other information observed on cephalometric radiographs (Table 1). In this case, extraction was performed for decrowding, shifting the teeth and mildly changing the facial profile. ${ }^{8}$ After aligning the anterior teeth, stripping of interproximal enamel could gain approximately $3 \mathrm{~mm}$ from canine to canine in the same arch. ${ }^{9}$ This approach not only solved the problem of crowded teeth, but also maintained the stability of the arch owing to the more contact between the teeth.

The decision of extraction sites is a key judgment in designing orthodontic treatment procedures. While the orthodontists are considering the need for tooth extraction, they must decide which tooth. As this case presented the mandibular asymmetry was associated with a shift to the right, so we extracted \#45, but not \#44 and \#34 of the opposite side, for dental midline correction. ${ }^{10}$ With regard to the anterior dental crowding, the crowding was relieved by tying the teeth strictly to ISW owing to its remarkable shape memory effect. Elastic chains were used to move the teeth and to align anterior teeth midline. The closed coil springs with different strengths were also used for teeth alignment. For the treatment of upper anterior teeth retraction in this case, $100 \mathrm{gf}$ was used on the right side while $50 \mathrm{gf}$ on the left side to correct the upper midline shift

Table 1: Pretreatment and post-treatment cephalometric data

$\begin{array}{lrrrc} & \text { Value } & \text { After } & \text { Mean } & \text { SD } \\ \text { Facial angle } & 94.8 & 95.2 & 84.83 & 3.05 \\ \text { Convexity } & 8.0 & 7.3 & 7.58 & 4.95 \\ \text { A-B plane } & -5.8 & -4.7 & -4.81 & 3.50 \\ \text { Mandibular plane } & 21.8 & 22.8 & 28.81 & 5.23 \\ \text { Y-axis } & 58.6 & 58.5 & 65.38 & 5.63 \\ & & & & \\ \text { Occlusal plane } & 4.8 & 2.8 & 11.42 & 3.64 \\ \text { Interincisal } & 128.5 & 146.1 & 124.09 & 7.63 \\ \text { L-1 to Occlusal } & 16.4 & 8.3 & 23.84 & 5.28 \\ \text { L-1 to Mandibular } & 89.4 & 78.3 & 96.33 & 5.78 \\ \text { U-1 to A-P plane } & 5.9 & 4.5 & 8.92 & 1.88 \\ \text { FMIA } & 68.8 & 78.9 & 54.63 & 6.47 \\ & & & & \\ \text { FH to SN plane } & 11.9 & 11.9 & 6.19 & 2.89 \\ & & & & \\ \text { SNA } & 86.9 & 87.2 & 82.32 & 3.45 \\ \text { SNB } & 82.7 & 83.1 & 78.90 & 3.45 \\ \text { SNA-SNB diff. } & 4.2 & 4.1 & 3.39 & 1.77 \\ \text { U-1 to N-P plane } & 8.4 & 6.7 & 11.74 & 2.73 \\ & & & & \\ \text { U-1 to FH plane } & 120.3 & 112.7 & 111.13 & 5.54 \\ \text { U-1 to SN plane } & 108.4 & 100.9 & 104.54 & 5.55 \\ & & & & \\ \text { Gonial angle } & 115.2 & 122.2 & 122.23 & 4.61 \\ \text { Ramus inclination } & 86.6 & 80.6 & 87.07 & 4.40 \\ & & & & \end{array}$

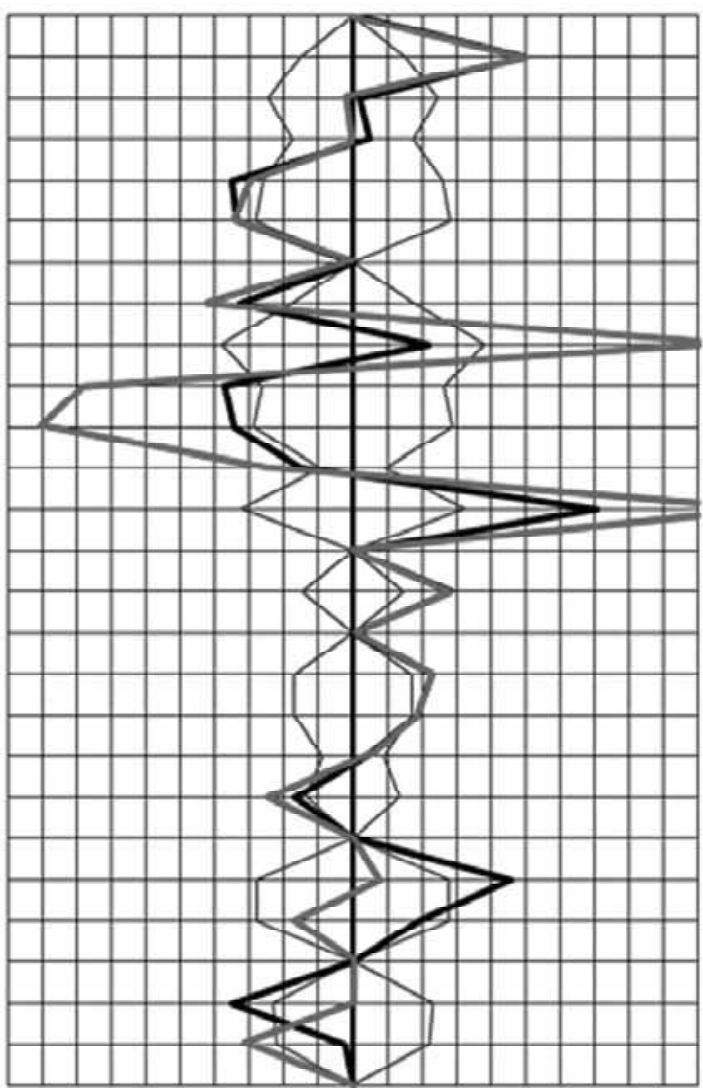


(Figs 1A to C). Because the first molar was erupting, IME was applied to add occlusal height for facilitating the rotation of mandible in the later stage of treatment. After the upper dental arch was shifted to the right side and lowered to the left side, the facial asymmetry was improved.

The facial profile from esthetic line was corrected by the extraction of teeth $\# 14, \# 24, \# 34, \# 45$ and anterior teeth. The management of a low mandibular plane angle of $21.8^{\circ}$ before active treatment resulted in a higher angle of $22.8^{\circ}$ after active treatment, which was contributed by the mechanism of pulling IME to lower the first molar (Table 1). Although the mandibular plane only slightly increased $1^{\circ}$, the lateral face was improved to a more straight profile on account of occlusal augmentation, the counterclockwise mandible, and reached overjet. ${ }^{11}$ The outcomes indicated that the crowns of upper central incisors are more tipping to palatal side, and the crowns of lower center incisors are also more tipping to lingual side, making the patient's face look more straight and pleasing ${ }^{12}$ (Table 1). Therefore, the positions of upper and lower lips were changed based on the tipping of anterior teeth (Fig. 6). With the use of intermaxillary elastics, the rotational movement of the mandible with left side shift could be measured (Fig. 6).

At the end of active orthodontic treatment, traditional retainers and dynamic positioner were delivered to maintain the improved intermaxillary relationship and to mildly remove the teeth as the teeth on the model was rearranged beforehand ${ }^{13}$ (Fig. 5). The use of dynamic positioner was important to accomplish the correction of facial asymmetry. The patient was asked to wear it for 24 hours in half a year, followed by 8 hours of wearing during sleep.

A more harmonious smile arc of the patient was achieved at the end of the treatment, owning to the corrections of anterior crowding teeth and facial asymmetry. As a result, the patient was satisfied with the treatment outcomes and pleased with her face appearance change.

\section{CONCLUSION}

Given the fact that the case had a facial asymmetry and concurrent maxillary and mandibular anterior dental crowding, it's difficult to achieve acceptable harmonization without using ISW that could fit individual physiological dental arch. With the use of ISW, an esthetic smiling arc was accomplished after the completion of orthodontic treatment and we gained patients' gratitude in return. However, the correction of facial asymmetry remains a major issue that needs more efforts to investigate. ${ }^{14}$

\section{REFERENCES}

1. Garrec P, Tavernier B, Jordan L. Evolution of flexural rigidity according to the cross-sectional dimension of a superelastic nickel titanium orthodontic wire. Eur J Orthod 2005;27:402-407.
2. Evans TJ, Durning P. Aligning archwires, the shape of things to come - a fourth and fifth phase of force delivery. Br J Orthod 1996;23:269-275.

3. Otsubo K. Development of the superelastic Ti-Ni alloy wire appropriate to the oral environment. J Jpn Orthod Soc 1994;53:641-650.

4. Melnik AK. A cephalometric study of mandibular asymmetry in a longitudinally followed sample of growing children. Am J Orthod Dentofacial Orthop 1992;101:355-366.

5. James PL, Treggiden R. Multiple neurofibromatosis associated with facial asymmetry. J Oral Surg 1975;33:439-442.

6. Gill DS, El Maaytah M, Naini FB. Risk factors for post-orthognathic condylar resorption: a review. World J Orthod 2008;9:21-25.

7. Ko EW, Huang CS, Chen YR. Characteristics and corrective outcome of face asymmetry by orthognathic surgery. J Oral Maxillofac Surg 2009;67:2201-2209.

8. Solomon MJ, English JD, Magness WB, McKee CJ. Long-term stability of lip bumper therapy followed by fixed appliances. Angle Orthod 2006;76:36-42.

9. Shu CL, Liu XQ, Wang GF. Effect of different orthodontic interproximal reduction methods on the enamel, Shanghai Kou Qiang Yi Xue 2012;21:384-388.

10. Yagi M, Ohno H, Takada K. Computational formulation of orthodontic tooth-extraction decisions. Part II: which tooth should be extracted? Angle Orthod 2009;79:892-898.

11. Rao SA, Thomas AM, Chopra S. Use of a modified anterior inclined plane in the treatment on the dentoskeletal Class II division 2 patient. J Indian Soc Pedod Prev Dent 2010;28:237-240.

12. Downarowicz P, Matthews-Brzozowska T, Kawala B, Drohomyretska M. Dynamic changes in morphometric analysis in patients following Class III bimaxillary surgery. Adv Clin Exp Med 2012;21:93-97.

13. Ohtsuka S, Kobayashi H, Kawamura T, Mori M, Shibasaki Y, Fukuhara T. Treatment of an adult Class III case using a dynamic positioner and its evaluation 5 years post-retention. Nihon Kyosei Shika Gakkai Zasshi 1986;45:503-510.

14. Tai K, Park JH, Ikeda K, Nishiyama A, Sato Y. Severe facial asymmetry and unilateral lingual crossbite treated with orthodontics and 2-jaw surgery: 5-year follow-up. Am J Orthod Dentofacial Orthop 2012;142:509-523.

\section{ABOUT THE AUTHORS}

\section{Chang Yuan-Chieh}

Resident, Department of Orthodontics and Dentistry, China Medical University Hospital, Taiwan

\section{YU Jian-Hong (Corresponding Author)}

Associate Professor, Department of Dentistry, College of Medicine China Medical University, Taiwan, e-mail: kenkoyu@mail.cmu.edu.tw

\section{Tsai Ya-Yu}

Resident, Department of Orthodontics and Dentistry, China Medical University Hospital, Taiwan

\section{YU Chien-Chih}

Associate Professor, Department of Pharmacy, College of Medicine China Medical University, Taiwan 